\title{
Phospholipid dynamics in ex vivo lung cancer and normal lung explants
}

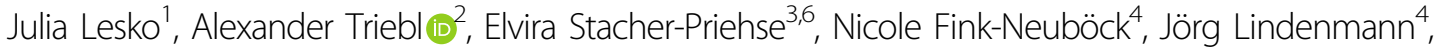 \\ Freyja-Maria Smolle-Jüttner ${ }^{4}$, Harald C. Köfeler ${ }^{2}$, Andelko Hrzenjak ${ }^{1,5}$, Horst Olschewski ${ }^{1,5}$ and Katharina Leithner (i) ${ }^{1}$
}

\begin{abstract}
In cancer cells, metabolic pathways are reprogrammed to promote cell proliferation and growth. While the rewiring of central biosynthetic pathways is being extensively studied, the dynamics of phospholipids in cancer cells are still poorly understood. In our study, we sought to evaluate de novo biosynthesis of glycerophospholipids (GPLs) in ex vivo lung cancer explants and corresponding normal lung tissue from six patients by utilizing a stable isotopic labeling approach. Incorporation of fully ${ }^{13} \mathrm{C}$-labeled glucose into the backbone of phosphatidylethanolamine (PE), phosphatidylcholine (PC), and phosphatidylinositol (PI) was analyzed by liquid chromatography/mass spectrometry. Lung cancer tissue showed significantly elevated isotopic enrichment within the glycerol backbone of $P E$, normalized to its incorporation into PI, compared to that in normal lung tissue; however, the size of the PE pool normalized to the size of the PI pool was smaller in tumor tissue. These findings indicate enhanced PE turnover in lung cancer tissue. Elevated biosynthesis of PE in lung cancer tissue was supported by enhanced expression of the PE biosynthesis genes ETNK2 and EPT1 and decreased expression of the PC and PI biosynthesis genes CHPT1 and CDS2, respectively, in different subtypes of lung cancer in publicly available datasets. Our study demonstrates that incorporation of glucosederived carbons into the glycerol backbone of GPLs can be monitored to study phospholipid dynamics in tumor explants and shows that PE turnover is elevated in lung cancer tissue compared to normal lung tissue.
\end{abstract}

\section{Introduction}

Lipogenesis, the de novo synthesis of lipids, is enhanced in many cancer types. Robust upregulation of fatty acid synthase (FASN) and other fatty acid biosynthetic genes has been noted in different cancers (for a review, see ref. ${ }^{1}$ ). However, fatty acid import may also be upregulated in cancer cells and contribute to tumor growth in a manner dependent on the mutational landscape and context ${ }^{2}$. The bulk of fatty acids produced in cancer cells is predominantly used to generate glycerophospholipids (GPLs), the major subtype of membrane phospholipids ${ }^{1,2}$. GPLs are important structural and functional components of biomembranes and play an important role in cell signaling and organelle function ${ }^{3}$. They

Correspondence: Katharina Leithner (katharina.leithner@medunigraz.at)

${ }^{1}$ Department of Internal Medicine, Division of Pulmonology, Medical University of Graz, 8036 Graz, Austria

${ }^{2}$ Core Facility Mass Spectrometry and Lipidomics, ZMF, Medical University of Graz, Graz, Austria

Full list of author information is available at the end of the article are structurally composed of a glycerol backbone linked to two fatty acid chains and different head groups, which define the GPL subclasses, for example, phosphatidylcholine (PC), phosphatidylethanolamine (PE), and phosphatidylinositol (PI). GPL synthesis proceeds mostly via lysophosphatidic acid (LPA), which is formed from glycerol-3-phosphate by glycerol-3-phosphate acyltransferases. LPA is then converted to phosphatidic acid (PA) by the addition of a second fatty acid chain. PA is dephosphorylated to generate diacylglycerol (DG), a precursor of PC or PE biosynthesis. Alternatively, PA is modified to produce cytidine diphosphate (CDP)-DG, a precursor of PI or cardiolipin biosynthesis ${ }^{3}$. In not only cancer cells but also normal proliferating cells, GPLs are continuously synthesized de novo, and their biosynthesis is balanced by their degradation ${ }^{4,5}$.

Levels of the PC and PE precursors phosphocholine and phosphoethanolamine have been shown to be increased in different cancers compared to the corresponding nonmalignant tissues ${ }^{6}$. In the past decade, progress in the 
Table 1 Patients' clinical data.

\begin{tabular}{|c|c|c|c|c|c|c|}
\hline Patient & $\# 1$ & $\# 2$ & \#3 & $\# 4$ & \#5 & \#6 \\
\hline Gender & M & $\mathrm{F}$ & $\mathrm{F}$ & $\mathrm{F}$ & M & $\mathrm{F}$ \\
\hline Age at surgery (years) & 57 & 74 & 58 & 75 & 54 & 83 \\
\hline Histology & LUAD & LUAD & LUAD & LUAD & LCNEM & LUAD \\
\hline Postop TNM & pT3 N1 M0 & pT2a NO MO & pT3 pN1 M0 & pT2b NO MO & pT2a NO MO & pT2a NO MO \\
\hline Stage & IIIA & $\mathrm{IB}$ & IIIA & $\| A$ & IB & IB \\
\hline Grade & G3 & G2 & G2 & G2 & G3 & $\mathrm{G} 2$ \\
\hline Explant culture & non-st & non-st & non-st & st & st & non-st; st \\
\hline
\end{tabular}

LUAD lung adenocarcinoma, LCNEM large-cell carcinoma with neuroendocrine morphology, postop TNM post-operative TNM classification (7th edition), non-st nonstarvation, st starvation.

knowledge of the phospholipidome under normal or pathological conditions has been fostered by the development of novel mass spectrometry techniques and analytical platforms, including electrospray ionization mass spectrometry approaches ${ }^{7-9}$. Intriguingly, comparative lipidomics showed marked differences in the composition of GPL in cancer tissues and the corresponding normal tissues (for reviews, see refs. ${ }^{7,8}$ ). In non-small cell lung cancer (NSCLC), GPL profiles were shown to clearly discriminate between tumor and normal lung tissue ${ }^{10,11}$. A mouse model of mutant epidermal growth factor receptor-driven lung cancer recently showed complex alterations in lipid profiles, including decreases in different PI species and a saturated PE species (PE 16:0), while the contents of certain triglycerides and phosphatidylmethanol species were increased compared to those in normal lung tissue ${ }^{12}$. While GPL profiling appears to be a highly useful tool for discriminating between normal and cancer tissue, even in intraoperative settings ${ }^{7,8}$, the contribution of GPL synthesis and degradation to cancer biology remains incompletely understood. Lipin-1, one of the enzymes responsible for the conversion of PA to DG, was shown to be upregulated in basal-like triple-negative breast cancer, and silencing lipin-1 significantly inhibited tumor growth in a breast cancer model in vivo ${ }^{13}$. Lipin-1 expression was shown to be elevated in NSCLC compared to normal lung tissue, and silencing lipin-1 in NSCLC cells induced endoplasmic reticulum stress and reduced proliferation and survival $^{14}$. While genetic manipulation of GPL biosynthetic genes provides valuable insights into their contributions to tumor growth, stable isotope tracing is needed to study the biosynthesis and degradation of different GPL classes in tumor tissues ${ }^{15}$. Here, we studied the relative dynamics of different GPL classes using ${ }^{13} \mathrm{C}$-labeled glucose in human NSCLC-derived explants.

\section{Materials and methods Lung cancer explants}

Samples of tumor tissue and corresponding noninvolved normal lung tissue from six consecutive patients with NSCLC who were referred for surgical resection to the Division of Thoracic and Hyperbaric Surgery, Medical University of Graz, were included in the study Patients treated with preoperative chemotherapy were excluded from the study. Patient- and tumor-specific data are listed in Table 1. Surgical specimens were cut into small fragments using a razor blade immediately after surgery, and the fragments (explants) were incubated in 6-well plates (up to six fragments per well) in DMEM (Dulbecco's modified Eagle's medium; Gibco, Waltham, MA) supplemented with the different concentrations of labeled or unlabeled glucose and serum described below for up to $72 \mathrm{~h}$ and were then subjected to liquid chromatography with tandem mass spectrometry (LC-MS/MS) analysis, viability analysis or fixation and paraffin embedding, as described below. The mean duration between surgical removal and the start of explant culture was $2.5 \mathrm{~h}$. Pathological diagnoses and the presence of tumor cells in the explants were confirmed by a pathologist (E.S.-P.). One patient had to be excluded since the tumor explants lacked tumor cells despite the initial diagnosis. The longest and shortest diameters of the tumor and lung explants from three patients were measured at the end of the experiments using an inverted microscope (Olympus Basic IX51, Olympus, Shinjuku, Tokyo, Japan). The size of the tumor explants (median largest diameter, $1.25 \mathrm{~mm}$; range, $0.75-2.0 \mathrm{~mm}$ ) was slightly smaller than that of the lung explants (median largest diameter, $1.82 \mathrm{~mm}$; range, $1.0-2.6 \mathrm{~mm}$ ). The study protocol was approved by the ethics review board of the Medical University of Graz. Signed informed consent was obtained from all patients prior to surgery.

\section{Stable isotope labeling}

After removal and dissection, tumors and lung explants were cultured in DMEM (Gibco, Waltham, MA) supplemented with different concentrations of labeled or unlabeled glucose, $1 \mathrm{mM}$ glutamine (Sigma-Aldrich, St. Louis, MO, USA), $100 \mathrm{U} / \mathrm{ml}$ penicillin and $100 \mu \mathrm{g} / \mathrm{ml}$ 
streptomycin (Gibco), with or without dialyzed fetal calf serum (FCS). For culture under "nonstarvation" conditions, media were supplemented with $5 \mathrm{mM}$ glucose (Sigma) or $\left[{ }^{13} \mathrm{C}_{6}\right]$ glucose (Cambridge Isotope Laboratories, Tewksbury, MA, USA) and $10 \%$ dialyzed FCS (Gibco). For culture under "starvation" conditions, media were supplemented with $1 \mathrm{mM}$ glucose (Sigma) or $\left[{ }^{13} \mathrm{C}_{6}\right]$ glucose (Cambridge Isotope Laboratories) without FCS. Media were replaced every $24 \mathrm{~h}$.

\section{Sample preparation for MS}

Lipid extraction was performed as described ${ }^{16}$ with minor modifications. Explants were briefly washed with glucose- and glutamine-free medium and were then transferred to $1.5 \mathrm{ml}$ of ice-cold methanol and homogenized with an Ultra-Turrax tissue homogenizer prior to three pulses of sonication for $10 \mathrm{~s}$. PC 24:0 (5 nmol) was added as an extraction control. After the addition of $2.5 \mathrm{ml}$ of methyl-tert-butyl ether (MTBE), the mixture was vortexed for $10 \mathrm{~s}$ and sonicated for $10 \mathrm{~min}$. After further addition of $2.5 \mathrm{ml}$ of MTBE and $10 \mathrm{~min}$ of shaking, $1.25 \mathrm{ml}$ of deionized water was added, and the mixture was subjected to $10 \mathrm{~min}$ of overhead shaking. Thereafter, the samples were centrifuged $\left(3 \mathrm{~min}\right.$ at $1350 \times g, 21^{\circ} \mathrm{C}$ ), and the upper organic phase was stored. The lower aqueous phase was reextracted with $2 \mathrm{ml}$ of the upper phase of MTBE/methanol/water (10/3/2.5, v/v/v). The resulting upper phases were combined and evaporated in a vacuum centrifuge. The dried lipids were resuspended in $1 \mathrm{ml}$ of chloroform/methanol $(1 / 1, \mathrm{v} / \mathrm{v})$.

\section{Hydrophilic interaction liquid chromatography/MS (HILIC/} MS) for analysis of isotopic enrichment of GPL

Chromatography was performed as described ${ }^{16}$. Briefly, the separation was performed on a Phenomenex Kinetex HILIC column $(2.1 \mathrm{~mm} \times 100 \mathrm{~mm}, 2.6 \mu \mathrm{m})$ (Phenomenex, Aschaffenburg, Germany) thermostatted to $50{ }^{\circ} \mathrm{C}$ in a Thermo Accela 1250 HPLC System (Thermo Fisher Scientific, Waltham, MA). Mobile phase A comprised deionized water containing $1 \%(\mathrm{v} / \mathrm{v})$ aqueous ammonium formate $(1 \mathrm{M})$ and $0.1 \%(\mathrm{v} / \mathrm{v})$ formic acid. Acetonitrile/ isopropanol $(5 / 2, \mathrm{v} / \mathrm{v})$ with the same additives was used as mobile phase B. Gradient elution started at $95 \%$ mobile phase B and was decreased to $75 \%$ mobile phase B over $10 \mathrm{~min}$. The column was reequilibrated for $8 \mathrm{~min}$ with $95 \%$ B. The flow rate was set to $250 \mu \mathrm{l} / \mathrm{min}$. Two microliters of sample, thermostatted to $10^{\circ} \mathrm{C}$, was injected using full loop injection mode.

An Orbitrap Velos Pro mass spectrometer (Thermo Fisher Scientific) was operated in negative ion mode alternating between full MS and tandem MS modes. Full scan spectra were acquired at a resolution of 30,000. Tandem mass spectra were acquired at a resolution of 7500. Phospholipid classes were analyzed using higher- energy collisional dissociation activation (normalized collision energy, 75; activation time, $0.1 \mathrm{~ms}$; FT first mass mode, fixed at mass-to-charge $(\mathrm{m} / \mathrm{z}) \mathrm{100})$. For the first $3 \mathrm{~min}$, PI molecular species were isolated $(\mathrm{m} / z 887.5 \pm 75)$; for the next $3 \mathrm{~min}$, PE molecular species $(\mathrm{m} / z 745.5 \pm 75)$; and for the remainder of the gradient, PC molecular species $(m / z \quad 804.5 \pm 100)$. Xcalibur Quan Browser and Microsoft Excel were used for data processing. For each lipid class investigated (PI, PE, and PC), two sets of fragment ions were analyzed: one set of head group fragment ions containing glycerol and one set of fragments not containing glycerol. For each set, the extracted ion chromatograms of five isotopologues (containing zero, one, two, three, or four ${ }^{13} \mathrm{C}$ atoms) were integrated using Lipid Data Analyzer ${ }^{17}$. The chemical structures of the target fragments have been reported in ref. ${ }^{16}$. The exact $\mathrm{m} / \mathrm{z}$ values are as follows: PC containing glycerol, 224.07; PC not containing glycerol, 168.04; PE containing glycerol, 196.04; PE not containing glycerol, 140.01; PI containing glycerol, 297.04; and PI not containing glycerol, 241.01. The areas of the isotopologues were normalized to the area of the corresponding monoisotopic ion. The isotopologue abundance was corrected for the natural abundance of ${ }^{13} \mathrm{C}$ using IsoCor software ${ }^{18}$. The normalized total pool sizes were determined by calculating the ratio of the PE area to the PI or PC area.

\section{MTS assay}

An MTS Cell Proliferation Colorimetric Assay Kit (Biovision, Milpitas, CA, USA) was used to assess explant viability according to the manufacturer's instructions. This method is based on the reduction of MTS tetrazolium caused by viable cells to yield a soluble, colored formazan product. Briefly, $20 \mu \mathrm{l}$ of MTS reagent (Biovision) was added to phenol red-free culture medium supplemented with 10\% dialyzed fetal bovine serum (Gibco) and antibiotics (Gibco). Fragments were incubated in MTScontaining medium for $3 \mathrm{~h}$ in 96 -well plates. Then, the fragments were washed in PBS, patted gently to remove excess the medium, and weighed separately. The optical density (OD) of the medium was analyzed in a spectrophotometer (SPECTRAmax PLUS 384, Molecular Devices, Sunnyvale, CA, USA) and normalized to the weight of each fragment. Tumor and lung fragments treated with $70 \%$ ethanol for $30 \mathrm{~min}$ were used as a negative control.

\section{Gene expression analysis using publicly available datasets}

Gene expression data of GPL biosynthetic genes were retrieved from the TCGA (The Cancer Genome Atlas) lung adenocarcinoma (LUAD) and lung squamous cell carcinoma (LUSC) datasets using the UCSC Xena platform (https://xenabrowser.net/). TCGA gene expression data were generated by the TCGA Research Network (https://www.cancer.gov/tcga). 


\section{Results}

\section{Determination of lung and tumor explant viability}

In solid tumors, blood perfusion is frequently inadequate, and steep local gradients for glucose and other nutrients are created ${ }^{19}$. On the other hand, the availability of nutrients is an important determinant of cancer cell metabolism ${ }^{20,21}$. Thus, we aimed to study cancer metabolism in human NSCLC explants under conditions of normal fasting blood levels of glucose $(5 \mathrm{mM})$ in the presence of serum ("nonstarvation conditions") or under conditions of $1 \mathrm{mM}$ glucose in the absence of serum ("starvation conditions"). First, we assessed the morphology of the lung and NSCLC explants cultured in the respective media. The architecture of the freshly isolated tissue and the cultured explants was similar, although signs of decreased viability were noted after $72 \mathrm{~h}$ of culture (Fig. 1a). Lung fragments showed moderate destruction of the alveolar structure and focally slightly thickened septa. Tumor fragments exhibited variable degrees of necrotic changes, which were more pronounced under starvation, but no explant was entirely necrotic (Fig. 1a). Tumor explants contained separated tumor cells and tumor cell nests and a large number of stromal cells and some lymphocytes. To assess lung and tumor explant viability under starvation conditions, we performed MTS assays. The weight-corrected OD values at 24,48 , and $72 \mathrm{~h}$ of culture were not significantly different from the value at $0 \mathrm{~h}$ (Fig. 1b).

\section{Contribution of de novo GPL synthesis to GPL pools}

We utilized fully ${ }^{13} \mathrm{C}$-labeled glucose under starvation and nonstarvation conditions to assess the contribution of de novo GPL synthesis to the total GPL abundance in lung and NSCLC explants. The duration of labeling was $72 \mathrm{~h}$, and the medium was changed daily. The labeled glucose was metabolized to glycerol-3-phosphate, which is required for the formation of the glycerol backbone of GPL (scheme depicted in Fig. 2a). Fragments of GPL backbones either containing or not containing glycerol were measured, and the relative isotopologue abundance values were calculated. Figure $2 \mathrm{~b}$ shows a mass spectrum of the different PC fragments. All three GPL classes analyzed, PC, PE, and PI, showed incorporation of the ${ }^{13} \mathrm{C}$ label into the glycerol backbone in the lung as well as NSCLC tissue (Fig. 2c, d). PC and PI showed the highest isotopologue enrichment values of up to $40 \%$ in the glycerol moiety (i.e., $40 \%$ of the PC and PI pools were derived from de novo biosynthesis by acylation of fatty acids to GPL precursors containing labeled glycerol). As expected, the glycerol-containing fragments were either unlabeled or contained an $\mathrm{M}+3$ label, corresponding to fully ${ }^{13} \mathrm{C}$-labeled glycerol (Fig. 2c, d). No label transfer from glucose to GPL fragments containing GPL head groups but not glycerol was found (Supplementary Fig. S1). Glucose is also a precursor for acetyl-CoA, which is required for fatty acid biosynthesis.

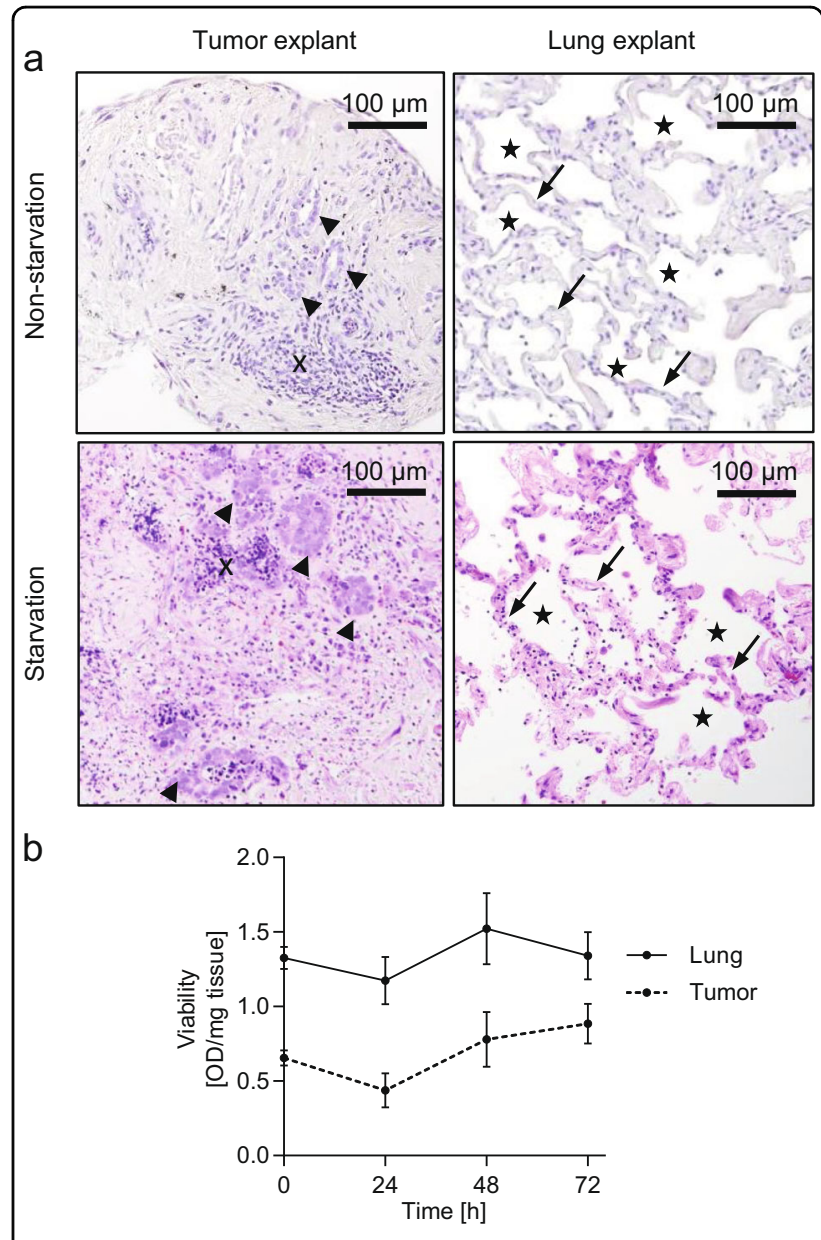

Fig. 1 Morphological appearance and viability of lung and tumor explants. a Hematoxylin and eosin staining of the tumor and the corresponding normal lung explants after $72 \mathrm{~h}$ of culture. An example of lung adenocarcinoma explants cultured in nonstarvation medium containing $5 \mathrm{mM}$ glucose and 10\% dialyzed FCS (upper row) and an example of a large-cell neuroendocrine carcinoma cultured in starvation medium containing $1 \mathrm{mM}$ glucose without FCS (lower row) are shown. Arrowheads, tumor cell nests; $x$, lymphocytes; arrows, alveolar walls; stars, alveolar spaces. b Viability of normal lung and tumor explants cultured under starvation conditions for different time intervals. Weight-corrected OD values are shown as the mean \pm SEM values. At least $n=10$ explants from a total of three patients were analyzed at each time point. No significant difference between viability immediately after dissection $(\mathrm{O} h)$ and at later timepoints was found.

However, fatty acids may also be imported from (serumcontaining) medium or recycled from preexisting phospholipids for de novo GPL biosynthesis. Thus, we did not assess label transfer to the fatty acid moieties of GPL to determine GPL turnover.

\section{Enhanced contribution of de novo PE biosynthesis to the total PE pool in tumors}

Unlabeled GPL-glycerol fragments were derived from either preexisting GPL not subjected to turnover or de 


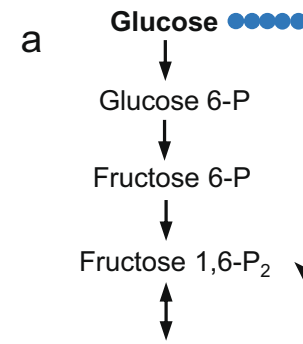

Glyceraldehyde 3-P

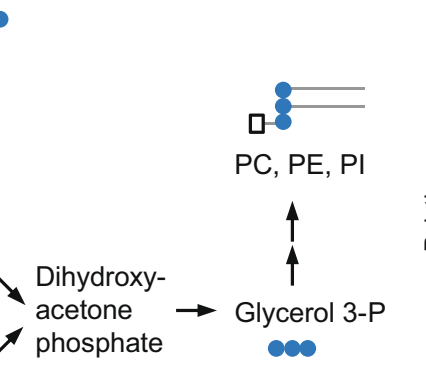

\section{b}

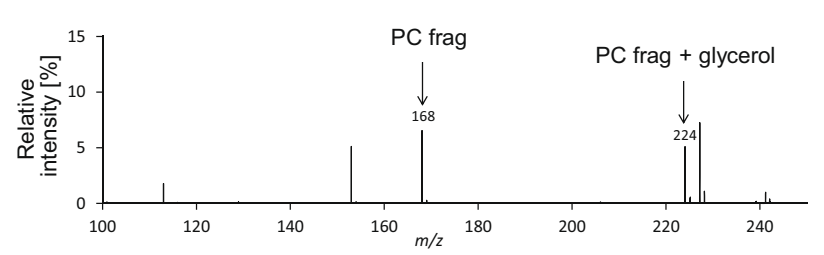

C
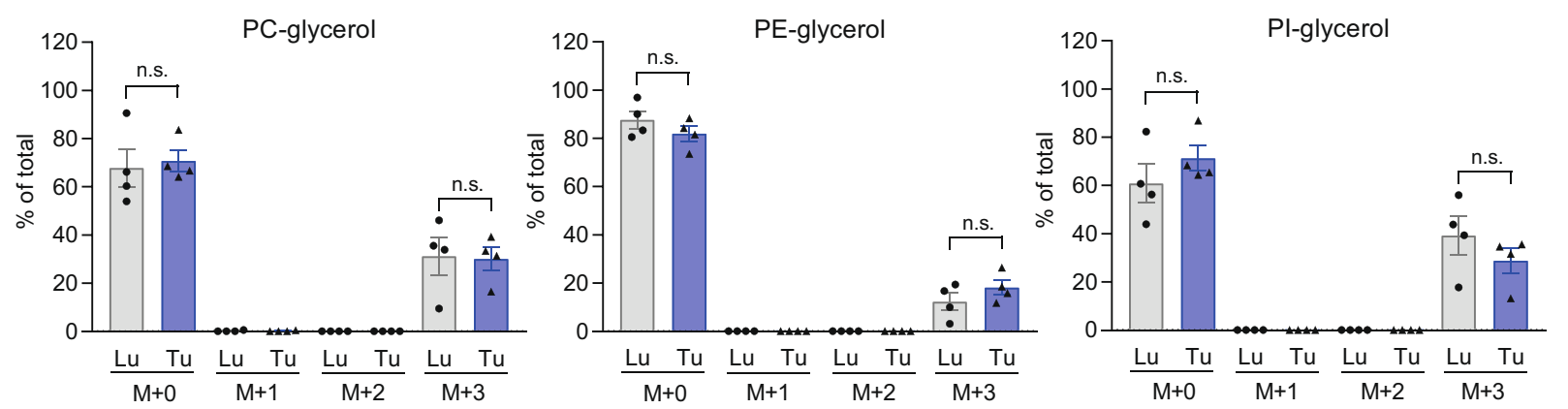

d
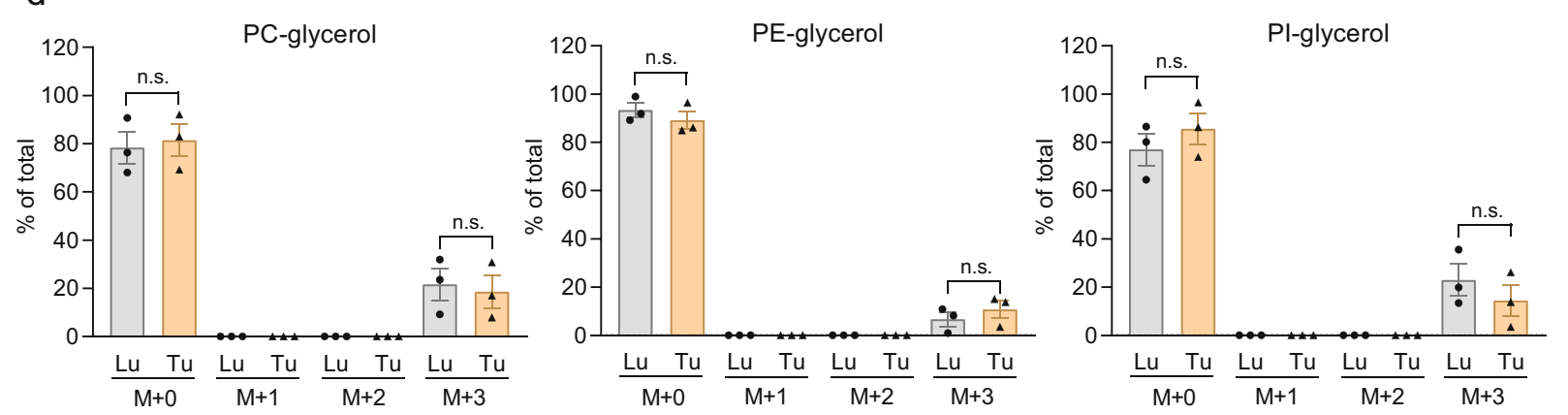

Fig. 2 Abundances of GPL-glycerol isotopologues in lung and tumor explants. a Metabolic pathway for the generation of phospholipid glycerol backbone from glucose. b Mass spectrum showing signal intensities at different mass-to-charge $(\mathrm{m} / \mathrm{z})$ ratios. The phosphatidylcholine (PC) head group fragment ion not containing glycerol (PC frag) and the corresponding fragment containing glycerol (PC frag + glycerol) are highlighted. c, d After $72 \mathrm{~h}$ of culture with $\left[{ }^{13} \mathrm{C}_{6}\right]$ glucose under either nonstarvation conditions (c) or starvation conditions (d), explants were analyzed by LC-MS/ MS, and the isotopologue abundances of GPLs containing labeled or unlabeled glycerol moieties was determined. M+ 0 denotes unlabeled glycerol-containing fragments, while $\mathrm{M}+3$ denotes fully ${ }^{13} \mathrm{C}$-labeled glycerol. No $\mathrm{M}+1$ or $\mathrm{M}+2$ (partially labeled glycerol) fragments were found. Natural abundance-corrected data from four (c) or three (d) patients are shown as the mean \pm SEM values. PC phosphatidylcholine, PE phosphatidylethanolamine, PI phosphatidylinositol, Lu lung, Tu tumor, n.s. nonsignificant.

novo synthesized GPL formed from unlabeled glycerol-3phosphate. To account for possible differences in glucose uptake and glycerol-3-phosphate labeling in the different tissues, we normalized the abundance of the $\mathrm{M}+3 \mathrm{PE}-$ glycerol isotopologue to that of $\mathrm{M}+3 \mathrm{PI}$-glycerol or $\mathrm{M}+$ 3 PC-glycerol. Under both experimental conditions, that is, nonstarvation and starvation, tumor explants displayed markedly elevated PE/PI enrichment ratios compared with those of normal lung explants (Fig. 3a, b). A similar but nonsignificant difference was found in the PE/PC ratios (Fig. 3a, b). The enhanced PE labeling was not affected by the level of glucose or serum in the labeling medium. Interestingly, the relative sizes of GPL pools were deregulated in the opposite direction in tumor tissue compared to normal lung tissue (Fig. 3c, d). In tumor tissue, the ratio of total PE to PI was significantly decreased under both experimental conditions (Fig. 3c, d). In summary, the larger contribution of de novo synthesis to the PE abundance in tumors and the smaller pool size suggests a relatively higher rate of $\mathrm{PE}$ turnover and a lower rate of PI turnover in lung cancer than in normal lung tissue. 


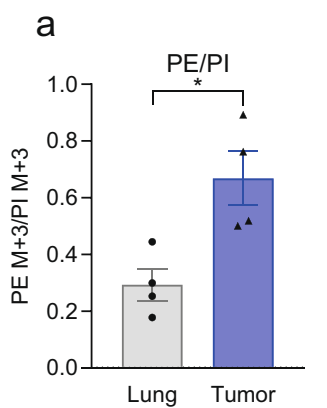

b

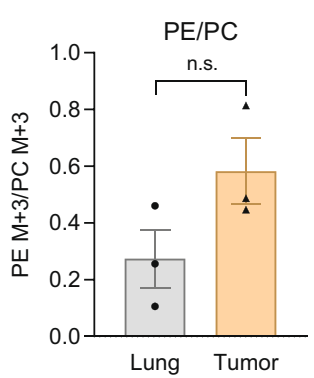

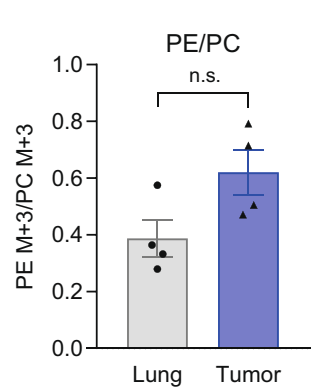

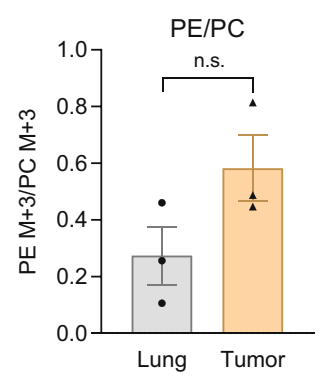

C
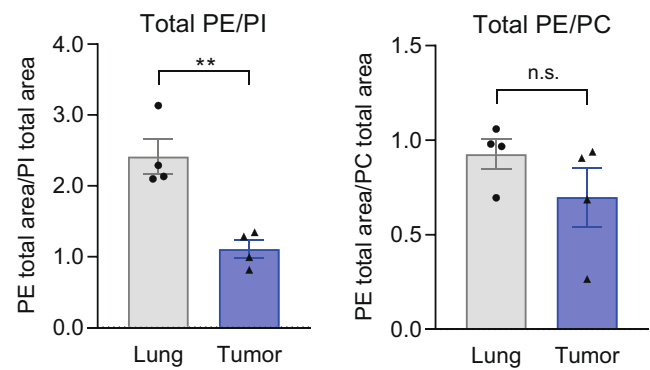

$\mathrm{d}$

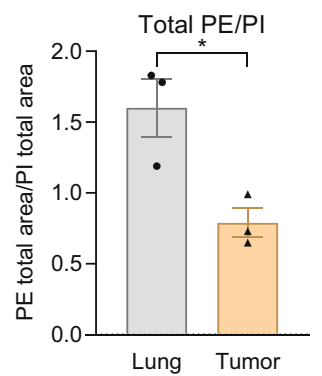

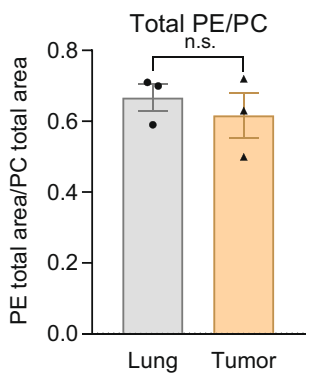

Fig. 3 Contribution of de novo synthesis to GPL pools during ex vivo culture of lung and tumor explants. After $72 \mathrm{~h}$ of culture with $\left[{ }^{13} \mathrm{C}_{6}\right]$ glucose under either nonstarvation conditions (a) or starvation conditions (b), explants were analyzed by LC-MS/MS, and the isotopologue abundance of PE containing ${ }^{13} \mathrm{C}$-labeled glycerol moieties was determined and normalized to the abundance of PI or PC to account for possible differences in precursor enrichment. $\mathrm{M}+0$ denotes unlabeled glycerol-containing fragments, while $\mathrm{M}+3$ denotes fully ${ }^{13} \mathrm{C}$-labeled glycerol. Relative abundances of $\mathrm{PE}, \mathrm{Pl}$, and $\mathrm{PC}$. $\mathbf{c}$, d Relative total abundance (labeled and unlabeled isotopologues) of PE normalized to $\mathrm{Pl}$ or $\mathrm{PC}$ in $\left[{ }^{13} \mathrm{C}_{6}\right]$ glucosetreated cells cultured under nonstarvation (c) or starvation conditions $(\mathbf{d})$. Data from four $(\mathbf{a}, \mathbf{c})$ or three $(\mathbf{b}$, $\mathbf{d})$ patients are shown as the mean $\pm \mathrm{SEM}$ values. PC phosphatidylcholine, PE phosphatidylethanolamine, PI phosphatidylinositol. ${ }^{*} P<0.05$ and ${ }^{*} P<0.01$ by two-tailed Student's $t$ test; n.S., nonsignificant.

PE biosynthesis genes are upregulated in NSCLC compared to normal lung tissue and are associated with poor survival

PE can be synthesized in mammalian cells via the CDPethanolamine pathway, by base exchange pathways from phosphatidylserine (PS) or PC, or by acylation of lyso-PE. Only the CDP-ethanolamine pathway represents true de novo synthesis ${ }^{22}$. The biosynthesis of CDP-ethanolamine starts with phosphorylation of ethanolamine by ethanolamine kinase (ETNK1 or ETNK2; scheme depicted in Fig. $4 c)^{22}$. Phosphoethanolamine is then converted to CDPethanolamine by ethanolamine-phosphate cytidylyltransferase (encoded by $P C Y T 2)^{22}$. The final step, the transfer of phosphoethanolamine from CDPethanolamine to DG, is catalyzed either by choline/ethanolamine phosphotransferase 1 (CEPT1), which has dual activity toward both CDP-ethanolamine in the PE biosynthesis pathway and CDP-choline in the PC biosynthesis pathway, or by the recently discovered selenoenzyme ethanolamine phosphotransferase 1 (EPT1, also referred to as SELENOI) ${ }^{22}$. The main pathway for the biosynthesis of $\mathrm{PC}$ is the Kennedy (CDP-choline) pathway. In this pathway, choline is phosphorylated by choline kinase (CHKA or $\mathrm{CHKB}$ ) to form phosphocholine. Phosphocholine is then metabolized to CDP-choline and finally transferred to DG by the choline phosphotransferases CHPT1 (also known as CPT1) and CEPT1, which is also responsible for PE biosynthesis ${ }^{23}$. Unlike PC and PE, PI is synthesized from CDP-DG ${ }^{3}$. CDP-DG synthase (CDS), with its two isoforms CDS1 and CDS2, activates PA to form the intermediate CDP-DG, which is then converted by PI synthase to PI. PA can be synthesized de novo or generated at the plasma membrane by the action of phospholipase $C$, which leads to hydrolysis of phosphatidylinositol $(4,5)$ bisphosphate, producing DG. DG is then rapidly phosphorylated to $\mathrm{PA}^{24}$. CDS1 and CDS2 are also the starting points for the synthesis of other, less abundant GPLs, including cardiolipins ${ }^{3}$.

We analyzed the expression of PE, PC, and PI biosynthetic genes in publicly available TCGA datasets of LUAD and LUSC - the two main subtypes of NSCLC - and in normal lung tissue. ETNK2, an ethanolamine kinase, and EPT1, the enzyme mediating the final step of PE biosynthesis, were significantly upregulated in lung adenocarcinoma (Fig. 4a) and in squamous cell carcinoma (Supplementary Fig. S2). ETNK1 was either not differentially expressed between tumor and lung tissue (adenocarcinoma) or showed slightly lower expression in tumor than in lung tissue (squamous cell carcinoma). PCYT2, 


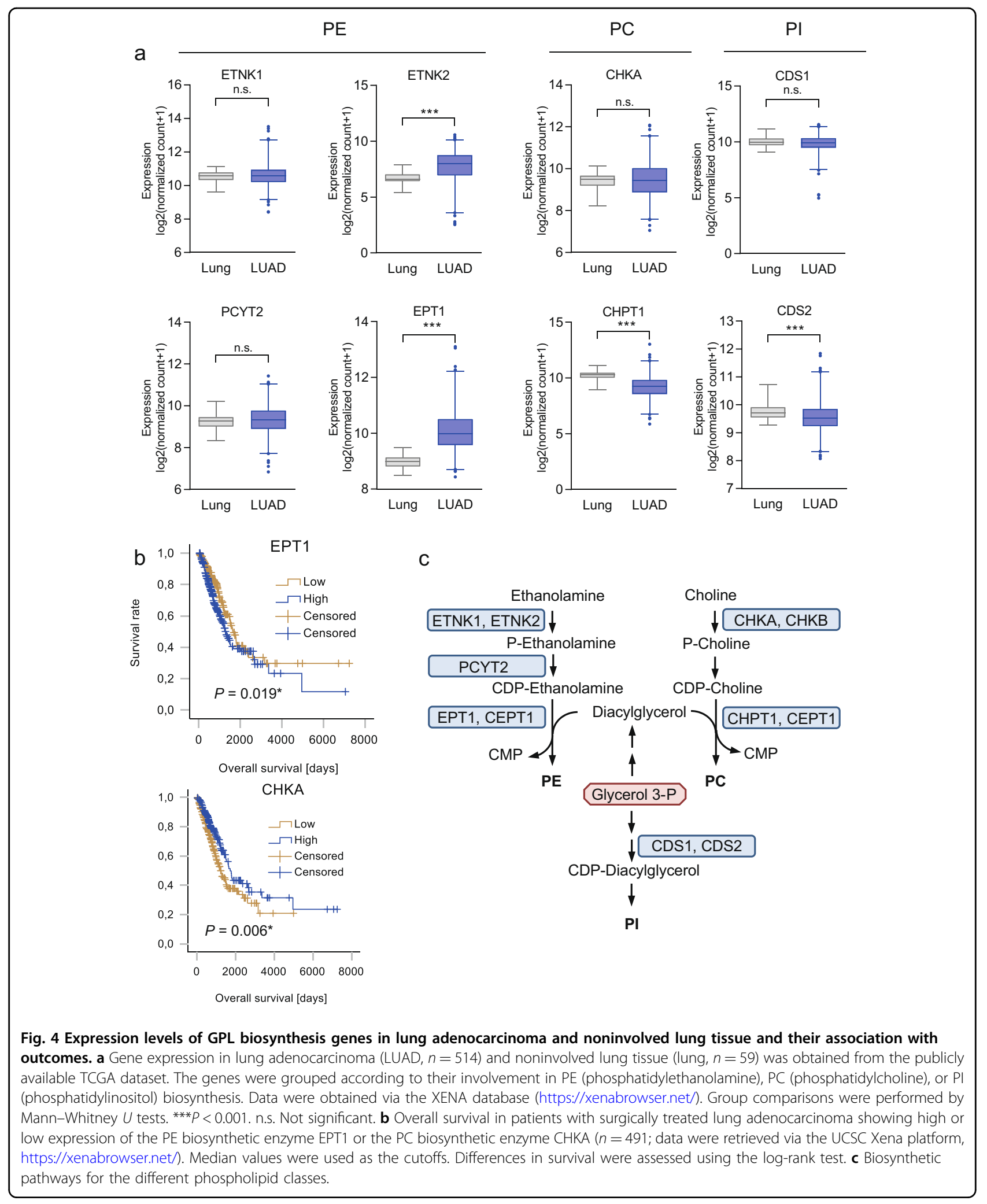

responsible for activation of phosphoethanolamine, was significantly upregulated in squamous cell carcinoma (Supplementary Fig. S2). In contrast, the PC biosynthesis gene CHPT1 was significantly downregulated in both NSCLC subtypes (Fig. 4a and Supplementary Fig. S2). Similarly, the PI biosynthetic genes CDS1 and CDS2 were 
downregulated in squamous cell carcinoma, and CDS2 was downregulated in adenocarcinoma compared to normal lung tissue (Fig. 4a, Supplementary Fig. S2). Importantly, ETNK2 and EPT1 exclusively mediate the biosynthesis of PE, in contrast to CHKB and CEPT1, which mediate the corresponding steps in $\mathrm{PE}$ biosynthesis but are also involved in PC biosynthesis (Fig. 4c). In lung adenocarcinoma, high expression of EPT1 but low expression of CHKA, a choline kinase, was significantly associated with poor overall survival (Fig. 4b).

\section{Discussion}

Cancer cells undergo metabolic reprogramming to support the biosynthesis of precursors for biomass production $^{25,26}$. Identification of major biosynthetic pathways that are crucial for the maintenance of certain metabolite pools might reveal specific metabolic vulnerabilities of cancer cells ${ }^{25,26}$. To date, only a few studies have addressed the role of de novo GPL synthesis in lung cancer. In our study, we found an enhanced contribution of de novo GPL synthesis to PE pools in NSCLC compared to normal lung tissue. Moreover, we found enhanced expression of PE biosynthetic genes in different subtypes of NSCLC compared to normal lung tissue.

In this study, we sought to determine relative differences in GPL isotopic labeling in NSCLC and normal lung explants, focusing on three major GPL classes-PC, PE, and PI. The method used relies on labeling of the glycerol phosphate precursor in the cultured explants and on quantitative determination of the glycerol moiety in the different GPL classes by MS/MS using a previously established detection protocol ${ }^{16}$. A similar approach using stable isotope-labeled glucose was recently applied to label the glycerol phosphate precursor of the GPL backbone to infer changes in GPL dynamics ${ }^{27}$. The half-life of the glycerol moiety of membrane GPL was determined to be an average of $29 \mathrm{~h}$ in rats ${ }^{28}$, and it ranged from 2 to 20 days for different GPL classes in a Drosophila model ${ }^{27}$. The long half-lives of GPL reported in the literature, however, suggest that an isotopic steady state is not achieved during the 72-h labeling period. Under such non-steady-state labeling conditions, the increase in label enrichment is determined by the metabolic flux of label incorporation, on the one hand, and by the pool size, on the other hand ${ }^{29}$. In fact, the pool sizes were found to be inversely related to the fractional glycerol enrichment values (Fig. 3c, d). The observed faster incorporation of glycerol into PE (normalized to PI) in the tumors and the lower size of the PE pool (normalized to the PI pool) showed that the relative turnover of $\mathrm{PE}$ is higher in tumor than in normal lung tissue. Due to the inherent limitation in tissue availability, we did not perform enrichment measurements at different time points in the tumor and lung explants to accurately determine the half-lives of the different GPLs (i.e., increases in labeled GPLs and decreases in unlabeled GPLs).

PE is one of the most abundant classes of GPL in cell membranes $^{3}$. Due to its conic shape, it promotes membrane curvature formation. However, PE also plays a major role in the regulation of other cellular functions, including autophagy ${ }^{3}$. The results of our study suggest that the expression of PE synthesis genes is upregulated and PE turnover is enhanced in NSCLC relative to the biosynthesis dynamics of other GPL classes. In a mouse model of KRAS-driven lung adenocarcinoma and GPL synthesis genes, as well as FA synthesis genes were among the top upregulated genes, indicating that lipogenesis is enhanced during KRAS-driven carcinogenesis ${ }^{30}$. PE synthesis genes were not included in the array used in that study. In a liver cancer model, inhibition of PE synthesis by meclizine in combination with glycolysis inhibition reduced tumor growth ${ }^{31}$. The results of the latter study suggest an interplay between PE synthesis and starvation in cancer. In fact, PE plays an important role in autophagy. Autophagy, a process of self-digestion, ensures the degradation of damaged organelles in not only normal but also cancer cells and provides metabolites under starvation conditions ${ }^{32}$. Covalent binding of PE to LC3, a key autophagy mediator, is required for its activity ${ }^{32}$. De novo PE synthesis was recently shown to proceed despite a lack of glucose in starved lung cancer cells by activation of gluconeogenesis, which provides glycerol phosphate from noncarbohydrate precursors ${ }^{16}$. Thus, PE might be required in cancer cells, especially under conditions of nutrient limitation and autophagy. In our study, we did not find major differences in PE-glycerol tracing in media containing $5 \mathrm{mM}{ }^{13} \mathrm{C}$-glucose and $1 \mathrm{mM}{ }^{13} \mathrm{C}$-glucose. However, our results were obtained in NSCLC explants from different patients, since too little tumor material was available from some patients to study all experimental conditions. The impact of nutritional status on de novo PE synthesis and PE turnover in cancer should thus be addressed in future studies. De novo synthesis of all three studied GPL classes, PE, PC, and PI, continued during explant culture in NSCLC as well as in normal lung explants. Stable isotope enrichment in the glycerol moiety was increased in PE relative to PI in lung cancer compared to normal lung tissue. Although a similar trend was noted in the PE/PC ratio, a contribution of decreased PI biosynthesis rates in lung cancer cells or altered PI pool sizes cannot be excluded. Interestingly, the expression of CDS1, a PI biosynthetic enzyme, has been shown to be reduced by ZEB1, an important mediator of epithelial-tomesenchymal transition ${ }^{33}$. Interestingly, p53, an important tumor suppressor, has been shown to enhance the expression of CDS1 and CDS2 $2^{34}$.

Cancer cells are at risk of undergoing ferroptosis, a mode of cell death induced by iron-dependent lipid 
peroxidation, partially due to an elevated intracellular iron content $^{35}$. Polyunsaturated fatty acids are particularly prone to undergo peroxidation and trigger ferroptosis unless protected by glutathione peroxidase 4, a phospholipid peroxidase ${ }^{36,37}$. The predominant GPL responsible for ferroptosis induction is still controversial. Recently, PEs containing PUFAs have been suggested to drive ferroptosis ${ }^{38,39}$. Thus, enhanced PE biosynthesis in cancer cells might be required to balance PE damage due to peroxidation.

Despite these recently discovered links between PE biosynthesis and cell survival and homeostasis, the role of PE biosynthetic enzymes in cancer progression is unclear. Here, we found that the expression of several PE biosynthetic genes, including ETNK2 and EPT1, was highly increased in tissues from two different subtypes of NSCLC compared to normal lung tissues, while the expression of PC and PI biosynthetic genes was decreased. Moreover, high expression of EPT1 was associated with poor overall survival in adenocarcinoma. In a previous study, ETNK1 was found to be amplified in a subset of lung adenocarcinomas, and ETNK1 amplification was found to be correlated with poor prognosis ${ }^{40}$.

Cultured explants or slices derived from human tumors are increasingly being utilized as a tumor model that maintains cell diversity and tissue architecture (for reviews, see refs. ${ }^{41,42}$ ). In cancer metabolism studies, this model allows the use of a defined and flexible tracer and the delineation of metabolic activity in the target tissue without systemic influences ${ }^{20,41}$. Not only stromal cells, such as fibroblasts and endothelial cells, but also immune cells present in human tumor tissue, are present in explants ${ }^{43}$. An advantage of this model is the ability to study tumor metabolism in its physiological 3D context. The observed changes in GPL dynamics occurred in the bulk of the tumor. Whether PE biosynthesis occurs primarily in neoplastic cancer cells or in stromal cells cannot be inferred from the data. In fact, cancer-associated fibroblasts from colon cancers have been shown to display enhanced expression of FASN and to release increased amounts of triglycerides and different GPLs, which are taken up by colon cancer cells ${ }^{44}$. Alterations in GPL dynamics in the different cell types within a tumor, for example, neoplastic tumor cells and stromal cells, should thus be assessed in future studies. In conclusion, this study shows that the incorporation of stable isotopic tracer-derived carbons into the glycerol backbone of GPL can be monitored in tumor explants, providing important insights into phospholipid dynamics in cancer. Although the number of patients was limited in this exploratory study, the data show that PE turnover and the expression of PE biosynthesis genes are strongly elevated in lung cancer tissue compared to normal lung tissue.

\section{Acknowledgements}

We thank Alexandra Bertsch and Bettina Schwemberger for excellent technical assistance. The results published here are based, in part, on data generated by the TCGA Research Network: https://www.cancer.gov/tcga. The study was funded by the Austrian Science Fund (FWF, P 28692-B31 to K.L.) and by the Austrian Federal Ministry of Education, Science, and Research (grant number BMWFW-10.420/0005-WF/N/3C/2017).

\section{Author details}

${ }^{1}$ Department of Internal Medicine, Division of Pulmonology, Medical University of Graz, 8036 Graz, Austria. ${ }^{2}$ Core Facility Mass Spectrometry and Lipidomics, ZMF, Medical University of Graz, Graz, Austria. ${ }^{3}$ Institute of Pathology, Medical University of Graz, Graz, Austria. ${ }^{4}$ Division of Thoracic and Hyperbaric Surgery, Medical University of Graz, Graz, Austria. ${ }^{5}$ Ludwig Boltzmann Institute for Lung Vascular Research, Graz, Austria. ${ }^{6}$ Present address: Institute of Pathology,

Asklepios Clinic Munich-Gauting, Munich, Germany

Conflict of interest

The authors declare that they have no conflict of interest.

\section{Publisher's note}

Springer Nature remains neutral with regard to jurisdictional claims in published maps and institutional affiliations.

Supplementary information accompanies this paper at https://doi.org/ 10.1038/s12276-020-00547-X

Received: 18 August 2020 Revised: 22 October 2020 Accepted: 4 November 2020.

Published online: 6 January 2021

\section{References}

1. Menendez, J. A. \& Lupu, R. Fatty acid synthase and the lipogenic phenotype in cancer pathogenesis. Nat. Rev. Cancer 7, 763-777 (2007).

2. Peck, B. \& Schulze, A. Lipid metabolism at the nexus of diet and tumor microenvironment. Trends Cancer 5, 693-703 (2019).

3. Bohdanowicz, M. \& Grinstein, S. Role of phospholipids in endocytosis, phagocytosis, and macropinocytosis. Physiol. Rev. 93, 69-106 (2013).

4. Jackowski, S. Coordination of membrane phospholipid synthesis with the cell cycle. J. Biol. Chem. 269, 3858-3867 (1994).

5. Zhang, X. H. et al. Disruption of G1-phase phospholipid turnover by inhibition of $\mathrm{Ca} 2+$-independent phospholipase A2 induces a p53-dependent cell-cycle arrest in G1 phase. J. Cell. Sci. 119, 1005-1015 (2006).

6. Cheng, M., Bhujwalla, Z. M. \& Glunde, K. Targeting phospholipid metabolism in cancer. Front. Oncol. 6, 266 (2016).

7. Ifa, D. R. \& Eberlin, L. S. Ambient ionization mass spectrometry for cancer diagnosis and surgical margin evaluation. Clin. Chem. 62, 111-123 (2016).

8. Takats, Z., Strittmatter, N. \& McKenzie, J. S. Ambient mass spectrometry in cancer research. Adv. Cancer Res. 134, 231-256 (2017).

9. Züllig, T. \& Köfeler, H. C. High resolution mass spectrometry in lipidomics. Mass Spectrom. Rev. https://doi.org/10.1002/mas.21627 (2020).

10. Zhang, M. et al. Accurate classification of non-small cell lung cancer (NSCLC) pathology and mapping of EGFR mutation spatial distribution by ambient mass spectrometry imaging. Front. Oncol. 9, 804 (2019).

11. Wei, Y. et al. Tissue spray ionization mass spectrometry for rapid recognition of human lung squamous cell carcinoma. Sci. Rep. 5, 10077 (2015).

12. Breitkopf, S. B. et al. A relative quantitative positive/negative ion switching method for untargeted lipidomics via high resolution LC-MS/MS from any biological source. Metabolomics 13, https://doi.org/10.1007/s11306-016-1157-8 (2017).

13. He, J. et al. Lipin-1 regulation of phospholipid synthesis maintains endoplasmic reticulum homeostasis and is critical for triple-negative breast cancer cell survival. FASEB J. 31, 2893-2904 (2017).

14. Fan, X. et al. Lipin-1 determines lung cancer cell survival and chemotherapy sensitivity by regulation of endoplasmic reticulum homeostasis and autophagy. Cancer Med. 7, 2541-2554 (2018). 
15. Brandsma, J., Bailey, A. P., Koster, G., Gould, A. P. \& Postle, A. D. Stable isotope analysis of dynamic lipidomics. Biochim. Biophys. Acta Mol. Cell. Biol. Lipids 1862, 792-796 (2017).

16. Leithner, K. et al. The glycerol backbone of phospholipids derives from noncarbohydrate precursors in starved lung cancer cells. Proc. Natl Acad. Sci. USA 115, 6225-6230 (2018).

17. Hartler, J. et al. Deciphering lipid structures based on platform-independent decision rules. Nat. Methods 14, 1171-1174 (2017)

18. Millard, P. et al. IsoCor: isotope correction for high-resolution MS labeling experiments. Bioinformatics 35, 4484-4487 (2019).

19. Vaupel, P. Tumor microenvironmental physiology and its implications for radiation oncology. Semin. Radiat. Oncol. 14, 198-206 (2004).

20. Muir, A., Danai, L. V. \& Vander Heiden, M. G. Microenvironmental regulation of cancer cell metabolism: implications for experimental design and translational studies. Dis. Model. Mech. 11, https://doi.org/10.1242/dmm.035758 (2018).

21. Cantor, J. R. \& Sabatini, D. M. Cancer cell metabolism: one hallmark, many faces. Cancer Discov. 2, 881-898 (2012).

22. Vance, J. E. Historical perspective: phosphatidylserine and phosphatidylethanolamine from the 1800 s to the present. J. Lipid Res. 59, 923-944 (2018).

23. McMaster, C. R. From yeast to humans-roles of the Kennedy pathway for phosphatidylcholine synthesis. FEBS Lett. 592, 1256-1272 (2018).

24. Blunsom, N. J. \& Cockcroft, S. Phosphatidylinositol synthesis at the endoplasmic reticulum. Biochim. Biophys. Acta Mol. Cell. Biol. Lipids 1865, 158471 (2020).

25. DeBerardinis, R. J. \& Chandel, N. S. Fundamentals of cancer metabolism. Sci. Adv. 2, e1600200 (2016)

26. Pavlova, N. N. \& Thompson, C. B. The emerging hallmarks of cancer metabolism. Cell. Metab. 23, 27-47 (2016).

27. Schlame, M., Xu, Y., Erdjument-Bromage, H., Neubert, T. A. \& Ren, M. Lipidomewide (13)C flux analysis: a novel tool to estimate the turnover of lipids in organisms and cultures. J. Lipid Res. 61, 95-104 (2020).

28. Omura, T., Siekevitz, P. \& Palade, G. E. Turnover of constituents of the endoplasmic reticulum membranes of rat hepatocytes. J. Biol. Chem. 242 2389-2396 (1967).

29. Buescher, J. M. et al. A roadmap for interpreting (13)C metabolite labeling patterns from cells. Curr. Opin. Biotechnol. 34, 189-201 (2015).
30. Gouw, A. M. et al. Oncogene KRAS activates fatty acid synthase, resulting in specific ERK and lipid signatures associated with lung adenocarcinoma. Proc. Natl Acad. Sci. USA 114, 4300-4305 (2017).

31. Guan, Y. et al. The phosphatidylethanolamine biosynthesis pathway provides a new target for cancer chemotherapy. J. Hepatol. 72, 746-760 (2020).

32. Kimmelman, A. C. \& White, E. Autophagy and tumor metabolism. Cell. Metab. 25, 1037-1043 (2017)

33. Gemmill, R. M. et al. ZEB1-responsive genes in non-small cell lung cancer. Cancer Lett. 300, 66-78 (2011)

34. Li, M. et al. p53 cooperates with SIRT6 to regulate cardiolipin de novo biosynthesis. Cell. Death Dis. 9, 941-018-0984-0 (2018).

35. Hirschhorn, T. \& Stockwell, B. R. The development of the concept of ferroptosis. Free Radic. Biol. Med. 133, 130-143 (2019).

36. Yang, W. S. et al. Peroxidation of polyunsaturated fatty acids by lipoxygenases drives ferroptosis. Proc. Natl Acad. Sci. USA 113, E4966-E4975 (2016).

37. Rysman, E. et al. De novo lipogenesis protects cancer cells from free radicals and chemotherapeutics by promoting membrane lipid saturation. Cancer Res. 70, 8117-8126 (2010)

38. Kagan, V. E. et al. Oxidized arachidonic and adrenic PEs navigate cells to ferroptosis. Nat. Chem. Biol. 13, 81-90 (2017).

39. Lee, $H$. et al. Energy-stress-mediated AMPK activation inhibits ferroptosis. Nat Cell Biol. 22, 225-234 (2020)

40. Han, $X$. et al. Comprehensive profiling of gene copy number alterations predicts patient prognosis in resected stages I-III lung adenocarcinoma. Front. Oncol. 9, 556 (2019).

41. Lane, A. N., Higashi, R. M. \& Fan, T. W. Preclinical models for interrogating drug action in human cancers using Stable Isotope Resolved Metabolomics (SIRM). Metabolomics 12, https://doi.org/10.1007/s11306-016-1065-y (2016).

42. Powley, I. R. et al. Patient-derived explants (PDEs) as a powerful preclinical platform for anti-cancer drug and biomarker discovery. Br. J. Cancer 122 735-744 (2020).

43. Leithner, $\mathrm{K}$. et al. Hypoxia increases membrane metallo-endopeptidase expression in a novel lung cancer ex vivo model - role of tumor stroma cells. BMC Cancer 14, 40 (2014).

44. Gong, J. et al. Reprogramming of lipid metabolism in cancer-associated fibroblasts potentiates migration of colorectal cancer cells. Cell. Death Dis. 11, 267-020-2434-z (2020). 